\title{
Enhanced osseointegration of titanium implants in a rat model of osteoporosis using multilayer bone mesenchymal stem cell sheets
}

\author{
YAN DUAN $^{1 *}$, WEI MA $^{1 *}$, DEHUA LI $^{1}$, TONGFEI WANG ${ }^{2}$ and BAOLIN LIU ${ }^{1}$ \\ ${ }^{1}$ State Key Laboratory of Military Stomatology \& National Clinical Research Center for Oral Diseases \& Shaanxi Engineering \\ Research Center for Dental Materials and Advanced Manufacture, Department of Dental Implants, School of Stomatology, \\ Fourth Military Medical University, Xi'an, Shaanxi 710032; \\ ${ }^{2}$ Department of Oncology, Tangdu Hospital, Fourth Military Medical University, Xi'an, Shaanxi 710038, P.R. China
}

Received March 11, 2016; Accepted March 17, 2017

DOI: $10.3892 / \mathrm{etm} .2017 .5303$

\begin{abstract}
The present study aimed to investigate whether bone marrow-derived mesenchymal stem cell (BMSC) sheets combined with titanium implants enhanced implant osseointegration in an ovariectomized (OVX) rat model of osteoporosis. Sprague-Dawley rats were randomly assigned into a test group and control group. Allogenic BMSCs were collected from the rats, cultured and stored via cryopreservation. At 6 months post-ovariectomy, establishment of the OVX model was confirmed by micro-computed tomography (CT) measurements. BMSC sheets were subsequently layered and wrapped over titanium implants for implantation. Unmodified implants served as the control. At 8 weeks post-implantation, samples were observed by micro-CT reconstruction and histomorphometric evaluation. Micro-CT reconstruction identified a marked improvement in the surrounding bone volume following treatment, with data analyses indicating a significant increase in bone volume in the BMSC-implant group compared with the control implant group $(\mathrm{P}<0.05)$. In addition, histological staining identified new bone formation and an increased rate of bone-implant contact surrounding the BMSC-implant constructs. These results indicate that the use of BMSC sheets as a novel tissue engineering approach improves the osseointegration of titanium implants in an osteoporosis model. This method may expand the operative
\end{abstract}

Correspondence to: Professor Baolin Liu, State Key Laboratory of Military Stomatology \& National Clinical Research Center for Oral Diseases \& Shaanxi Engineering Research Center for Dental Materials and Advanced Manufacture, Department of Dental Implants, School of Stomatology, Fourth Military Medical University, 145 West Changle Road, Xi'an, Shaanxi 710032, P.R. China

E-mail: baolin@fmmu.edu.cn

*Contributed equally

Key words: bone mesenchymal stem cells, cell sheets, ovariectomized, implant indications in patients with osteoporosis and improve the success rate of clinical dental implant treatments.

\section{Introduction}

Osteoporosis is a progressive skeletal disorder characterized by reduced bone mineral density (BMD) and microarchitectural deterioration, which typically leads to increased bone fragility and susceptibility to fracture (1). In patients with osteoporosis, the differentiation, proliferation and osteogenic capabilities of bone marrow-derived mesenchymal stem cells (BMSCs) are inhibited during bone healing, which results in reduced bone formation and compromised osseointegration of bone implants $(2,3)$. In addition, the regulation of bone tissue regeneration and remodeling in the bone marrow, particularly regarding the quantity and quality of crucial biological factors, such as VEGF-A and TGF-beta1, is markedly altered in ageing animals (4). As a result, bone healing and regeneration around dental implants are substantially decreased in patients with osteoporosis, resulting in higher rates of implant failure (5-7). A previous study demonstrated that osseointegration in osteoporosis is associated with multiple complications, including bone graft failure, prolonged bone healing time and a high bone implant failure rate in clinical dentistry (8). Results of a retrospective clinical study have also suggested that certain general disorders, particularly osteoporosis, may contribute to implant failure following maxillary sinus bone grafting (9). However, histological analyses of osseointegrated implants removed from patients with osteoporosis have identified a close contact between healthy bone and the implant surface, with this bone-implant contact (BIC) confirming that osseointegration had been achieved $(10,11)$. These inconsistencies in previous results indicate that further experimental studies are warranted to determine the influence of osteoporosis on bone regeneration around implants.

Two established animal models currently exist for the study of osteoporosis, the OVX model and the immobilization-induced bone loss model. Neither of these animal models identically represent the stages of osteoporosis in humans (12); however, they do provide an experimental comparison. Small and large animals, including rats, rabbits and sheep, may be used for these models depending on which aspects of 
osteoporosis are being investigated. Among these models, the OVX rat model, with a short generation time and low cost, is the most popular animal model that has been validated to represent bone loss in women (13), particularly during the early stages of osteoporosis $(14,15)$. The OVX rat model exhibits significant loss of volume and strength in the vertebral cancellous bone as early as 3 months after ovariectomy; a relatively shorter generation period compared with larger animal models of osteoporosis $(16,17)$. In addition, the diameter of the rat tibia is typically $\sim 5 \mathrm{~mm}$, which is larger than the implant diameter of $2.6 \mathrm{~mm}$, thus leaving $>1 \mathrm{~mm}$ of surrounding bone to maintain the stability of the implant. For these reasons, the OVX rat model of osteoporosis was adopted in the present study.

Two methods are typically administered to treat patients with osteoporosis who exhibit impaired osteogenesis. The first aims to improve patients' osteogenic capability through systemic medicinal treatment of osteoporosis, and the second aims to improve the implant biocompatibility by modifying its surface. At present, treatment for osteoporosis primarily involves the systemic administration of medication. For example, bisphosphonates are used to improve osseous healing potential, possibly through effects on BMSC mitogenesis, and proproliferative and antiapoptotic effects on osteoblasts, though their underlying mechanisms of action remain unknown (18). In modern implant dentistry, studies have focused on the surface modification of implant materials, with the aim of improving the biocompatibility and in vivo performance of implants. In particular, studies have focused on various methods that alter the surface characteristics of implants, including modification of the implant surface roughness (19). However, it is difficult to improve implant osseointegration through physiochemical modifications alone (20), as bone healing and growth is a complicated process, involving migration, proliferation and differentiation of osteogenic cells.

Tissue engineering-based approaches have been documented to improve local osteogenesis around an implant. BMSCs are among the most commonly used cells in such approaches (21), due to their high differentiation potential (including osteogenic differentiation), proliferative ability and suitability for autologous transplantation due to their ability to avoid an immunologic reaction (22). In animal models of osteoporosis, dental implant modifications using cell-based tissue engineering techniques have demonstrated potential for the repair of bone defects (23). Previous studies by our group have evaluated an implant technique involving BMSC sheets, whereby the modified constructs were characterized by a higher cell density, greater content of extracellular matrix (ECM) and growth factors, the ability for facile harvesting without the need for chemical treatment and stability around the BIC (24). Our previous studies have also demonstrated that BMSC sheets may be used to create a BMSC-implant construct with osteogenic potential in vivo and in vitro (25). However, the ability of a BMSC-based tissue engineering approach to improve the osseointegration of dental implant materials in patients with osteoporosis remains unknown. Therefore, in the present study, a rat model of osteoporosis was used to evaluate the osseointegration of a BMSC sheet-titanium implant complex. The data obtained suggest that this novel BMSC sheet-based tissue engineering strategy may enhance bone regeneration around titanium implants.

\section{Materials and methods}

Preparation of implant samples. The surfaces of 60 polished titanium implants were rinsed in ethanol twice and distilled water twice. Then, an MJ2000 ultrasonic machine (Wuxi Meijie Ultrasonic Cleaning Equipment Co., Ltd., Wuxi, China) was used for deep cleaning of the implants.

Animal model preparation. A total of 40 female SpragueDawley rats (Medical Laboratory Animal Center, The Fourth Military Medical University, Xi'an, China; weight, 110 $\pm 8.73 \mathrm{~g}$; age, 10 weeks old) were used in the current study, according to institutional guidelines for the care of experimental animals of the Fourth Military Medical University (Xi'an, China). Animal experiments were performed according to an animal study protocol approved by the Ethics Committee of the Fourth Military Medical University (approval no. 2015065). The rats were housed individually in the cages with the room temperature $\sim 18-24^{\circ} \mathrm{C}$ and relative humidity between $40-60 \%$. Fluorescent lighting was provided on a 12-h light/dark cycle. Free access to tap water and standard rodent feed (CE-2; CLEA Japan, Inc., Tokyo, Japan) was given to all rats. Rats were randomly divided into the following two groups: An ovariectomized (OVX) group in which a bilateral ovariectomy was performed $(n=20)$; and a sham operation group $(n=10)$. After intramuscular injection of $1 \%$ pentobarbital $(20 \mathrm{mg} / \mathrm{kg})$, rats were under deep of anesthesia. Skin preparation and sterilization was performed and ophthalmic scissors were used to cut $\sim 2 \mathrm{~mm}$ at both sides of the rat dorsalis, exposing the psoas muscle layer. The psoas muscle was longitudinally cut to exposure the abdominal cavity $(1.5 \mathrm{~mm})$, exposing the bilateral ovaries attached with mesentery and ligation was performed. The same procedure was performed in the sham group without ligation after exposure. After the suture, iodophor was used to disinfect the incision area. The two groups of rats were housed under the conditions mentioned above. In the following surgical procedures, the BMSC-based implant was inserted into the right tibia (the test group); and the titanium control implant was inserted into the left tibia (the negative control group) of the same OVX rat. Two rats in the OVX group were excluded as they succumbed to anesthesia-related fatality. Therefore, a total 18 rats (36 samples) were analyzed in the present study.

BMSC isolation and culture. From the total Sprague-Dawley rats originally obtained from the Animal Experiment Center at the Fourth Military Medical University, 10 female rats (10 weeks old; weighing 80-120 g) were used from bone marrow harvesting. Approval for bone marrow harvesting was obtained from the Institutional Animal Care and Use Committee of the Fourth Military Medical University. Rat BMSCs were isolated and harvested as previously described (26). Briefly, rats were sacrificed by an overdose of pentobarbital $(1 \%, 100 \mathrm{mg} / \mathrm{kg})$ that was intraperitoneally injected. Then, bilateral tibiae and femora were dissected and tissue scissors were used to cut the ends of the long bones. Bone marrow was extruded into Dulbecco's modified Eagle medium (Gibco; Thermo Fisher Scientific, Inc., Waltham, MA, USA) using a syringe. After 
centrifugation (560 x g for $4 \mathrm{~min}$ at room temperature), $5 \times 10^{6}$ bone marrow cells were seeded into T25 flasks. Cells were incubated at $37^{\circ} \mathrm{C}$ with $5 \% \mathrm{CO}_{2}$ for $24 \mathrm{~h}$. Following incubation, DMEM supplemented with $1 \mathrm{ng} / \mathrm{ml} \mathrm{b}$-FGF was added to replenish and remove non-adherent cells. The medium was then replaced every 3 days. Cells were passaged after reaching $90 \%$ confluency. Cells from the fourth passage were used for experiments in the present study.

Construction of the osteoporosis rat model. No rats experienced complications during the 3-month post-operative recovery period and their weight continued to increase until the time of sacrifice. A total of 4 samples could not be included in the analysis as 2 rats in the OVX group died upon anesthesia induction. The remaining 36 samples were used for subsequent experiments.

Using micro-computed tomography (CT), reconstruction of the three-dimensional (3D) bone structure and related quantitative analysis may be performed based on selected images, and the bone density in live rats following ovariectomy may be measured. In the current study, the osteoporosis model produced by overiectomy in Sprague-Dawley rats was verified at 3 months post-operation by evaluation of BMD using micro-CT analysis.

Phenotypic analysis of rat BMSCs. Attached BMSCs were trypsinized, centrifuged $\left(560 \times \mathrm{g}\right.$ for $4 \mathrm{~min}$ at $\left.37^{\circ} \mathrm{C}\right)$ and stained with phycoerythrin-conjugated rat antibodies directed against CD-90, CD-45, CD-29 and CD-34 respectively (1:500; Cat. nos. 553014, 561588, 562154 and 551387; BD Biosciences, San Jose, CA, USA) for $30 \mathrm{~min}$ at room temperature. BMSCs were then rinsed twice with PBS. After centrifugation at $560 \mathrm{x}$ at room temperature, cells were re-suspended in $500 \mu \mathrm{l}$ PBS to a concentration of $\sim 5 \times 10^{5}$ cells $/ 100 \mu \mathrm{l}$. Cells incubated without antibodies served as controls. Flow cytometric analysis of cell surface protein expression was performed using an Epics XL flow cytometer with CXP Analysis Software 15 Network (Beckman Coulter, Inc., Brea, CA, USA), as described previously (27).

Multilineage differentiation potential of isolated BMSCs in vitro. To induce osteogenic differentiation, rat BMSCs were seeded into 6 -well plates at a density of $1 \times 10^{5}$ cells/well and cultured in $\alpha$-Minimum Essential Medium ( $\alpha$-MEM; Sigma-Aldrich; Merck KGaA Darmstadt, Germany) in an atmosphere containing $10 \% \mathrm{CO}_{2}$ at $37^{\circ} \mathrm{C}$ for $24 \mathrm{~h}$ until cells had adhered. Medium was then replenished with the following osteoinductive medium: $\alpha$-MEM supplemented with $10 \%$ fetal bovine serum (FBS), $0.1 \mathrm{mM}$ dexamethasone (Sigma-Aldrich; Merck KGaA), 50 mM ascorbate-2-phosphate (Sinopharm Chemical Reagent Co., Ltd., Shanghai, China), 10 mM $\beta$-glycerophosphate (Alfa Aesar; Thermo Fisher Scientific, Inc. Waltham, MA, USA), and 1\% penicillin-streptomycin-glutamine liquid (Gibco; Thermo Fisher Scientific, Inc.). $\alpha$-MEM medium was used as the control group. The osteoinductive medium was replaced every 3 days. After 10 days of culture $\left(10 \% \mathrm{CO}_{2}\right.$ at $\left.37^{\circ} \mathrm{C}\right)$, BMSCs in parallel wells were examined for mineralized nodule formation using light microscopy. Cells were fixed after 28 days of osteoinduction in $70 \%$ ethanol at room temperature for $15 \mathrm{~min}$ and then subjected to Alizarin
Red S staining ( $\mathrm{pH}$ 4; Sigma-Aldrich; Merck KGaA). Images were captured using an inverted phase-contrast microscope.

To induce adipogenic differentiation, rat BMSCs were seeded into 6 -well plates at a density of $1 \times 10^{5}$ cells/well for the induction of lipid formation in vitro. Cells were cultured in $\alpha$-MEM (Sigma-Aldrich; Merck KGaA) culture $\left(10 \% \mathrm{CO}_{2}\right.$ at $37^{\circ} \mathrm{C}$ ) until they reached $60 \%$ confluency. The medium was then replaced with the following adipogenic induction medium: $\alpha$-MEM supplemented with 10\% FBS, $200 \mathrm{mM}$ indomethacin, $10 \mathrm{mM}$ insulin, $1 \mathrm{mM}$ dexamethasone, $0.5 \mathrm{mM}$ isobutyl methylxanthine and $1 \%$ antibiotic/antimycotic solution $(10,000 \mathrm{U}$ penicillin and $10 \mathrm{mg}$ streptomycin; all Sigma-Aldrich; Merck KGaA). The adipogenic induction medium was refreshed every 2-3 days. After 21 days, the cells were fixed in $70 \%$ ethanol for $15 \mathrm{~min}$ at room temperature and stained with Oil Red O (Sigma-Aldrich; Merck KGaA) for 20 min. Images were captured using an inverted phase-contrast microscope.

Induction of BMSC sheet formation. Allogenic BMSCs were seeded into 6 -well plates at a density of $2 \times 10^{5}$ cells/well and cultured in osteoinductive medium for 4 weeks in an atmosphere containing $10 \% \mathrm{CO}_{2}$ at $37^{\circ} \mathrm{C}$ until cell layers had formed. Intact layers of BMSCs were then detached from the substratum using a cell scraper and forceps, and combined with other detached layers to form multilayer BMSC sheets. The BMSC sheets were wrapped tightly around titanium implants (6 $\mathrm{mm}$ in length, $2.6 \mathrm{~mm}$ in diameter, $0.2 \mathrm{~mm}$ thread depth and $0.5 \mathrm{~mm}$ thread pitch; Northwest Institute for Nonferrous Metal Research, Xi'an, China) that were specifically designed for the present study and the BMSC-implant constructs were placed in an incubator containing $10 \% \mathrm{CO}_{2}$ at $37^{\circ} \mathrm{C}$ for $1 \mathrm{~h}$ to enhance the stability of the construct prior to implantation into the tibiae of rats in the OVX and sham operation groups.

Implantation of the BMSC-implant constructs. In preparation for surgery, rats were anesthetized with an intraperitoneal injection of a mixed narcotic, consisting of xylazine $(5 \mathrm{mg} / \mathrm{kg}$; Bayer AG, Leverkusen, Germany) and ketamine (75 mg/kg; Pfizer, Inc., New York, NY, USA). The hind limbs were shaved and disinfected with lodophor (Mundipharma $\mathrm{GmbH}$, Limburg, Germany). A longitudinal medial incision was made on the skin. With the knee in flexion, a $2 \mathrm{~mm}$ diameter canal was drilled through the intercondylar notch of the femur using an implant drill (NobelReplace Tapered Surgery Kit; Nobel Biocare, Zürich, Switerland) and monitor (Nobel Biocare, Zürich, Switzerland). Each rat received an unmodified control titanium implant and a BMSC sheet-coated implant in the left and right tibiae, respectively. The skin and soft tissue were closed with a single subcuticular stitch (1-0 vicryl suture; Johnson \& Johnson Corp., Shanghai, China). Before the rats awoke, postoperative motion of the knee joint was verified.

BMD measurement. High-resolution micro-CT was performed using an Inveon Siemens micro-CT scanner (Siemens AG, Munich, Germany) in the small animal scanning mode $(80 \mathrm{kV}$; $500 \mathrm{~mA} ; 800 \mathrm{msec}$ integration time) to measure BMD $\left(\mathrm{mg} / \mathrm{cm}^{2}\right)$. Rats were anesthetized by intra-abdominal injection of pentobarbital sodium solution $(1 \%, 20 \mathrm{mg} / \mathrm{kg})$ and kept in a limited motion holder to ensure image clarity. BMD measurements 
A B

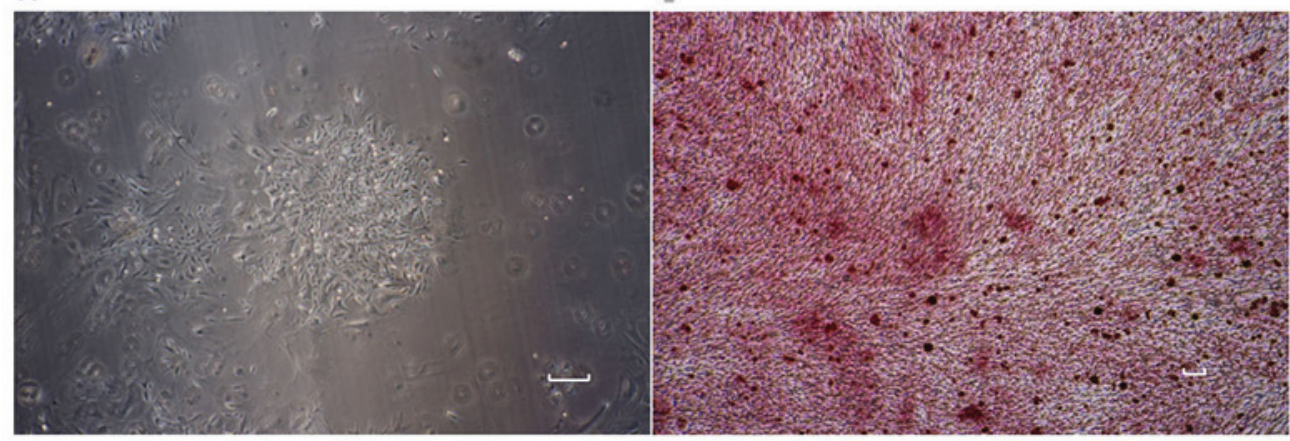

C

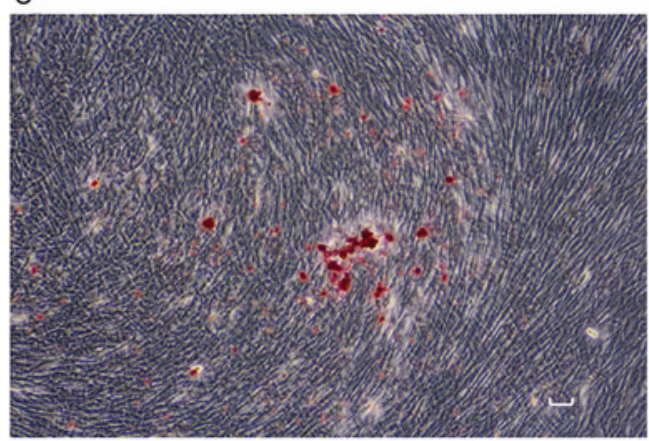

Figure 1. Proliferation and pluripotency of BMSCs. (A) BMSCs (magnification, $\mathrm{x} 40$ ) were isolated from 10-week-old Sprague-Dawley rats and the multilineage differentiation potential of cells was confirmed by inducing their differentiation into (B) osteogenic (Alizarin Red S staining; magnification, x10) and (C) adipogenic (Oil Red O staining; magnification, x10) lineages. Scale bar, $20 \mu \mathrm{m}$. BMSC, bone marrow-derived mesenchymal stem cells.
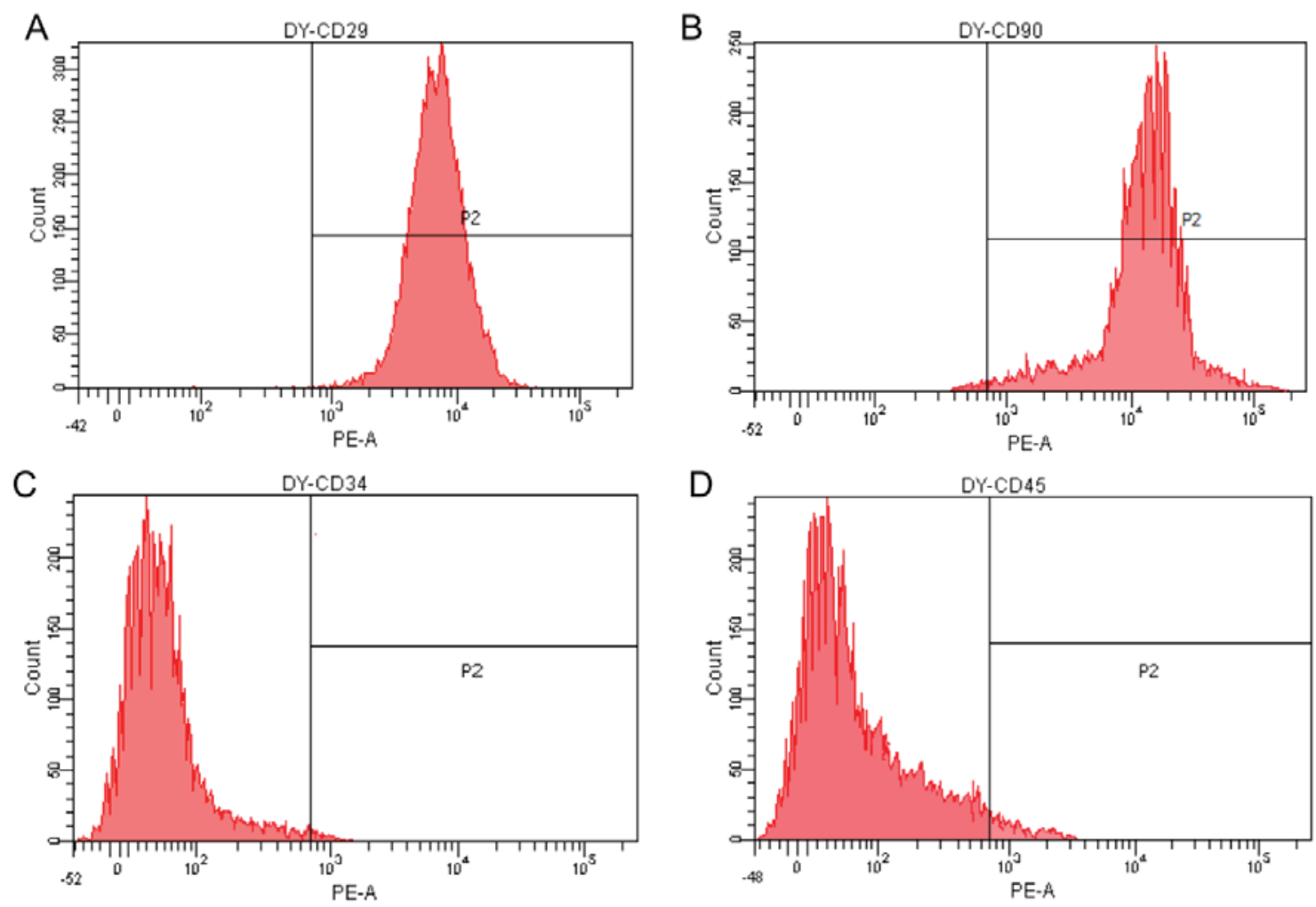

Figure 2. Flow cytometric analysis to identify rat BMSCs. The presence of rat BMSCs was confirmed by positive staining for (A) CD29 and (B) CD90 and negative staining for (C) CD34 and (D) CD45. BMSC, bone marrow-derived mesenchymal stem cells; CD, cluster of differentiation.

were also performed in live animals prior to implant surgery to identify the implant insertion area. Scans and analyses were conducted twice; 12 weeks after OVX animal model formation and 8 weeks after implantation (the implant healing period). 
Table I. Bone density indices in the control and OVX groups.

\begin{tabular}{lrr}
\hline Bone density index & Control & \multicolumn{1}{c}{ OVX } \\
\hline BV/TV, \% & $55.27 \pm 2.43$ & $9.78 \pm 0.06^{\mathrm{a}}$ \\
BS/BV, \% & $20.29 \pm 1.86$ & $23.25 \pm 0.57^{\mathrm{a}}$ \\
Tb.Th, mm & $0.99 \pm 0.25$ & $0.09 \pm 0.01^{\mathrm{a}}$ \\
Tb.N, mm & $5.85 \pm 0.36$ & $1.14 \pm 0.04^{\mathrm{a}}$ \\
Tb.sp, mm & $0.07 \pm 0.01$ & $0.78 \pm 0.05^{\mathrm{a}}$ \\
Tb.P.F, $1 / \mathrm{mm}$ & $5.81 \pm 0.08$ & $6.75 \pm 0.04^{\mathrm{a}}$
\end{tabular}

a $<<0.05$ vs. the control group. OVX, ovariectomized; BV/TV, bone volume/total volume; BS/BV, bone surface/bone volume; Tb.N, trabecular number; Tb.Sp, trabecular separation; Tb.Th, trabecular thickness.

Table II. Bone density indices at the bone-implant interface after 8 weeks of healing.

\begin{tabular}{lrc}
\hline Bone density index & Ti implant & BMSC-Ti construct \\
\hline BV/TV, \% & $12.82 \pm 0.07$ & $30.71 \pm 0.23^{\mathrm{a}}$ \\
BS/BV, \% & $10.25 \pm 0.07$ & $12.82 \pm 0.11$ \\
Tb.Th, mm & $0.09 \pm 0.01$ & $0.16 \pm 0.01^{\mathrm{a}}$ \\
Tb.N, mm & $1.14 \pm 0.05$ & $1.97 \pm 0.05$ \\
Tb.sp, mm & $0.79 \pm 0.06$ & $0.35 \pm 0.03^{\mathrm{a}}$ \\
Tb.P.F, $1 / \mathrm{mm}$ & $6.75 \pm 0.06$ & $3.46 \pm 0.03^{\mathrm{a}}$ \\
\hline
\end{tabular}

${ }^{\mathrm{a}} \mathrm{P}<0.05$ vs. the $\mathrm{Ti}$ implant group. Ti, titanium; BMSC, bone marrow-derived mesenchymal stem cells; BV/TV, bone volume/total volume; BS/BV, bone surface/bone volume; Tb.N, trabecular number; Tb.Sp, trabecular separation; Tb.Th, trabecular thickness.

Evaluation of BIC interfaces: Micro-CT reconstruction and measurement. The two rat groups were subjected to local micro-CT after 8 weeks of bone healing to evaluate hard tissue regeneration around the implants. A circle around the implant (5-mm in size) was chosen as the region of interest, and a 3D image was reconstructed and evaluated using Inveon Research Workplace software (version 2.2.0; Siemens AG). Specifically, the bone formation indices of trabecular thickness (Tb.Th), trabecular spacing (Tb.Sp), trabecular number (Tb.N), bone volume/total volume (BV/TV) and bone surface/bone volume (BS/BV) were measured.

Evaluation of bone-implant interfaces: Histological and histomorphometric analysis. After an implant healing period of 8 weeks, all rats were sacrificed with an intra-abdominal injection of $1 \%$ pentobarbital sodium solution $100 \mathrm{mg} / \mathrm{kg}$. The tibiae of rats were then harvested and fixed with $10 \%$ formalin solution ( $\mathrm{pH} \sim 7$ ) for 3 weeks at $4^{\circ} \mathrm{C}$ before being embedded in resin as a cylinder (diameter $20 \mathrm{~mm}$, height $40 \mathrm{~mm}$ ). Two sections $(\sim 120-\mu$ m thick) were made from every one sample. All sections were cut through the long axis of the implant using a LEICA SP1600 high-speed precision microtome (Leica Microsystems GmbH, Wetzlar, Germany), then polished to

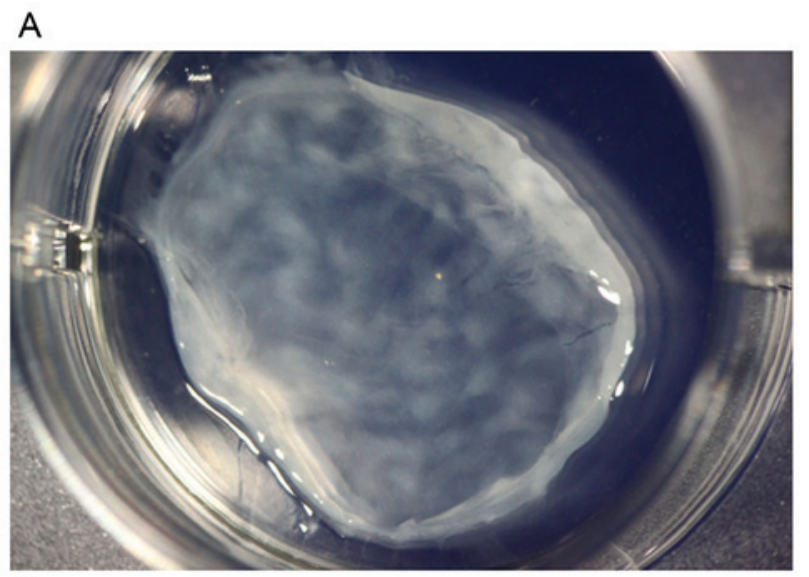

B

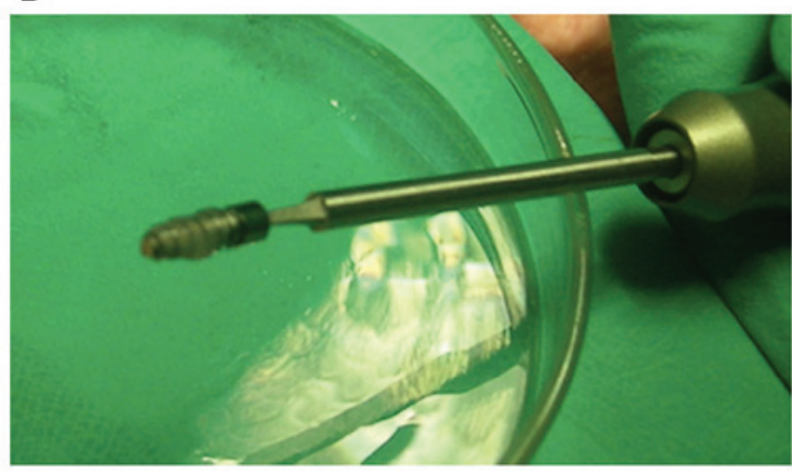

Figure 3. BMSC sheet formation and seeding. (A) BMSC sheets were formed over 7-10 days in osteoinductive medium and were seeded onto (B) titanium implants. BMSC, bone marrow-derived mesenchymal stem cells.

$100 \mu \mathrm{m}$ thickness for observation under a light microscope. One section was stained using Masson-Ponceau Tri-Chrome method and the other section from the same sample was stained by Van Gieson's method (28). A histomorphometric evaluation with a Leica Qwin Pro-Image Analysis system (Leica QWin Pro 16 system; Leica Microsystems GmbH) was used to quantify the image data. Specifically, the rate of BIC was measured for subsequent quantitative analysis of new bone formation.

Statistical analysis. All data are expressed as the mean \pm standard deviation. All statistical analyses were performed using SPSS (version 20.0; IBM SPSS, Armonk, NY, USA). Analysis of the variance were used, specifically, one-way ANOVA was performed on the micro-CT data and two-way ANOVA for $\mathrm{BIC}$ ratios in order to compare the differences between the two implant groups through the 8 -week healing period. $\mathrm{P}<0.05$ was considered to indicate a statistically significant difference.

\section{Results}

Phenotype and differentiation of the isolated rat BMSCs. Isolated rat BMSCs exhibited a polygonal shape and adhered to the bottom of culture flasks after $24 \mathrm{~h}$ of culture. The BMSCs proliferated in $\alpha$-MEM to reach a confluency of $80 \%$ in 7-10 days (Fig. 1A). Calcium nodes were stained by Alizarin Red (Fig. 1B). By contrast, Oil Red O staining of BMSCs revealed cellular lipids characteristic of adipocytes (Fig. 1C). 

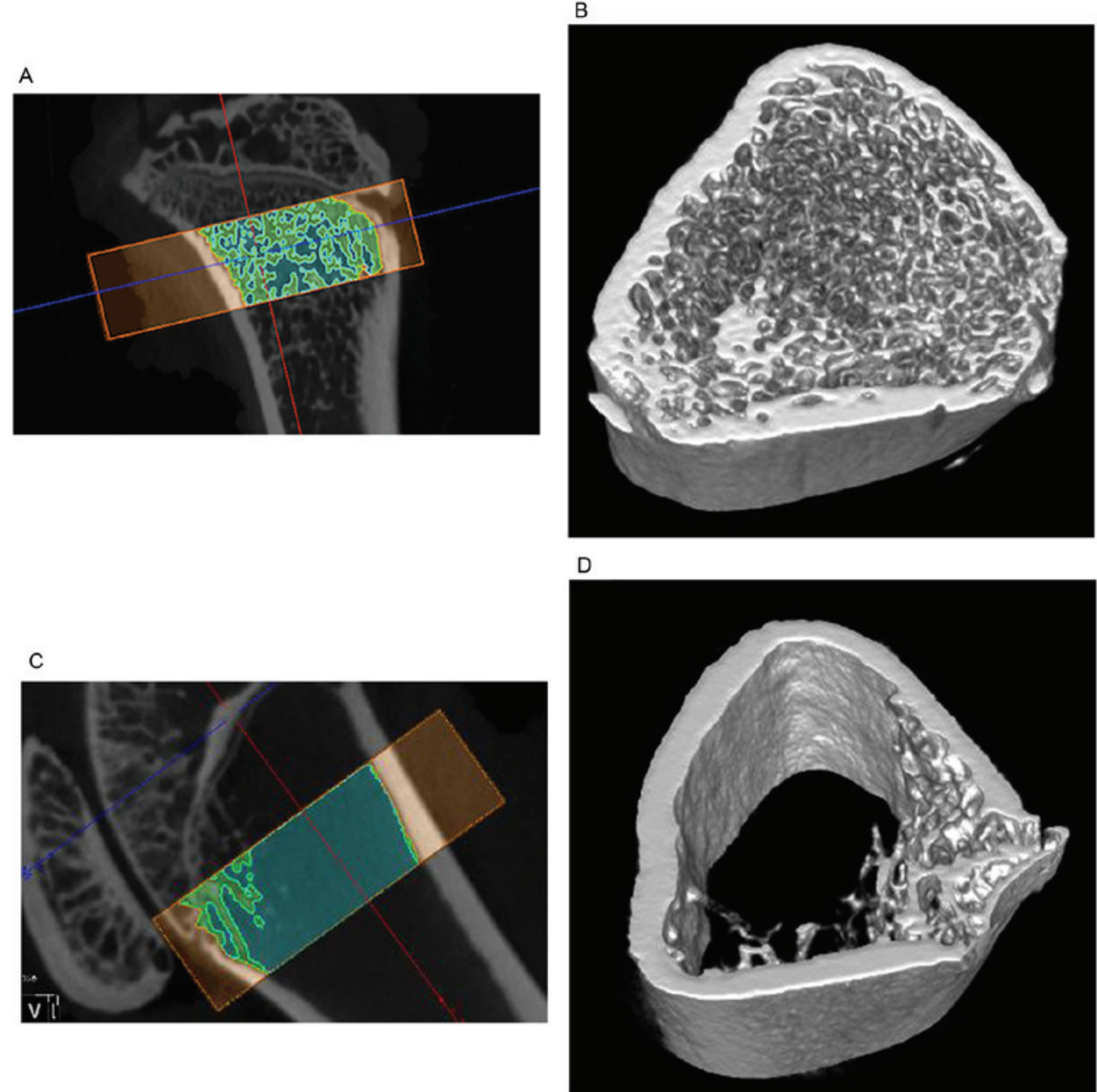

Figure 4. Micro-computed tomography reconstruction of the bone 3 months after ovariectomy. (A) Sagittal plane of the sham group (n=10). (B) horizontal plane of the sham group. The bone structure was complete with dense trabecular bone and continued cortical bone. (C) Sagittal plane of ovariectomized group $(n=18)$. (D) horizontal plane of the ovariectomized group. The bone structure was incomplete with sparse trabecular bone and discontinued cortical bone.

Prior to differentiation, expanded BMSCs were uniformly positive for the mesenchymal stem cell (MSC) markers CD29 and CD90, and negative for the hematopoietic lineage marker CD34 and leukocyte common antigen CD45 (Fig. 2).

Multilayer BMSC sheet formation and combining with titanium implants. After the culture in osteoinductive medium for 4 weeks, BMSCs reached over $90 \%$ confluency on the bottom of the culture dish and proliferated over the basal cell layer to form a high-density 3D sheet-like structure. Three layers of the cell sheets were combined and wrapped around titanium implants (Fig. 3).

Establishment of OVX model. As depicted in Table I, at 3 months after ovariectomy, the bone density index in the OVX group for BV/TV and Tb.Th were significantly decreased compared with the sham operation group, and significantly increased for Tb.sp. The micro CT analysis also show the loss of trabecular framework (Fig. 4).

Micro-CT evaluation of bone formation in vivo. Rats in the two groups were given an implant healing period of 8 weeks to allow the host bone tissue regeneration around the implants. The images obtained by micro-CT indicated that osseointegration of implants had occurred in both groups (Fig. 5). In the test group, newly formed bone surrounding the BMSC-implant construct (Fig. 5A and B) was greater in quality and quantity compared with that in the control group (Fig. 5C and D). In addition, more organized supporting bone and greater trabecular construction was observed around the BMSC-implant construct in the test group. The bone density index around the implants, BIC (Fig. 6), BV/TV, Tb.Th, Tb.Sp, Tb.N and $\mathrm{BS} / \mathrm{BV}$ were measured by micro CT analysis (Table II). The $\mathrm{BIC}, \mathrm{BV} / \mathrm{TV}$ and $\mathrm{Tb}$.Th of the BMSC-implant test group were significantly higher, while the Tb.Sp was significantly lower, compared with those in the control group (all $\mathrm{P}<0.05$ ). However, there was no significant difference in Tb.N and $\mathrm{BS} / \mathrm{BV}$ values between the two groups in this study, which was probably caused by insufficient sample volume.

Histological evaluation of bone formation in vivo. Following Masson-Ponceau Tri-Chrome and Van Gieson's staining, all the sections were evaluated by light microscopy (Fig. 7). At 8 weeks post-implantation, it was observed that bone 
A

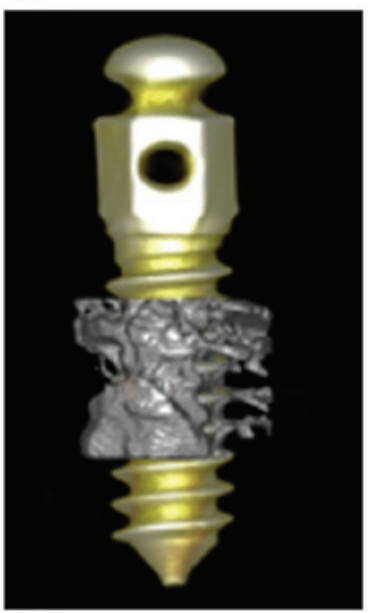

C

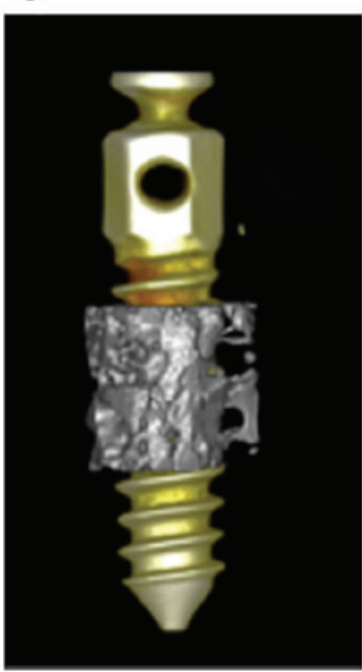

B

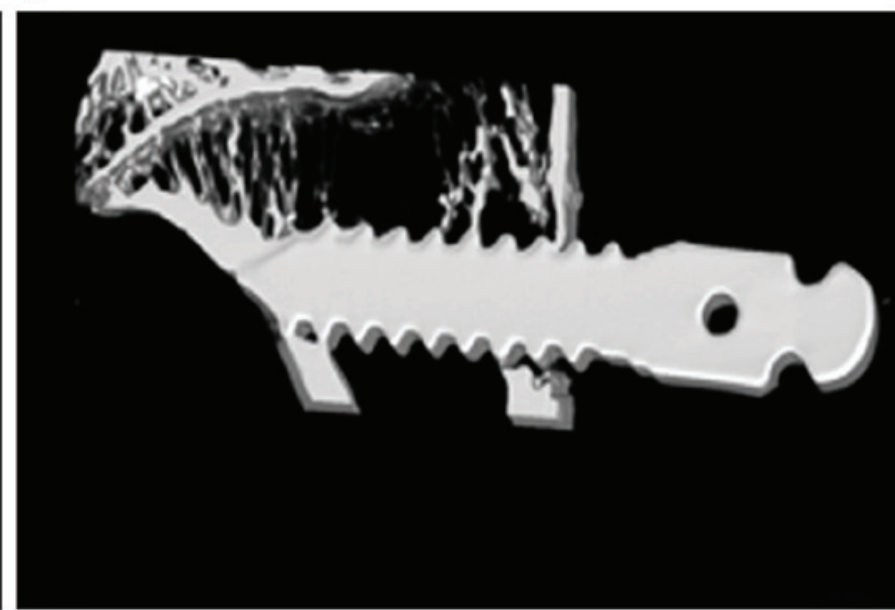

D

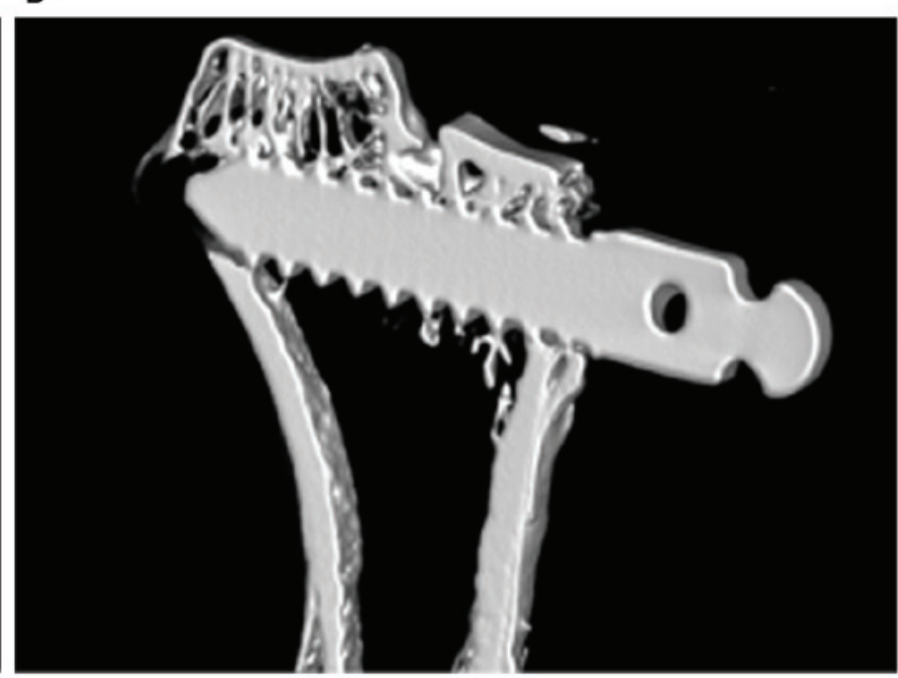

Figure 5. Micro-CT reconstruction of the bone-implant interface. (A) 3D reconstruction and (B) cross-sectional view of bone-implant interface in the test group. (C) 3D reconstruction and (D) cross-sectional view of bone-implant interface in the control group. After 8 weeks of bone healing, images from micro-CT reconstruction indicated that bone formation around the bone marrow-derived mesenchymal stem cells sheet-implant constructs was greater compared with the unmodified titanium implants.

regeneration occurred more rapidly in the test group, and newly formed bone around the BMSC-implant constructs exhibited a greater BMD compared with the control group. In addition, bone tissue was closely adhered to the BMSC-implant constructs and there was no apparent gap around the BMSC-implant constructs. Subsequent histomorphometric analysis of 18 BMSC-implant construct specimens indicated that the $\mathrm{BIC}$ ratio in the BMSC-implant group $(30.7 \pm 3.1 \%)$ was significantly higher compared with that in the control group $(16.8 \pm 3.9 \%)$ after 8 weeks of healing in vivo $(\mathrm{P}<0.001$; Fig. 6).

\section{Discussion}

Regarding dental implants, adequate osseointegration with the host bone tissue is of great importance and required for successful restoration. Scholars in implant dentistry have so far designed and evaluated a variety methods aimed at enhancing osseointegration, primarily by improving the speed and quality of bone regeneration (29). However, in medically compromised patients suffering from a systematic disease such as osteoporosis, diabetes and periodontal inflammation,

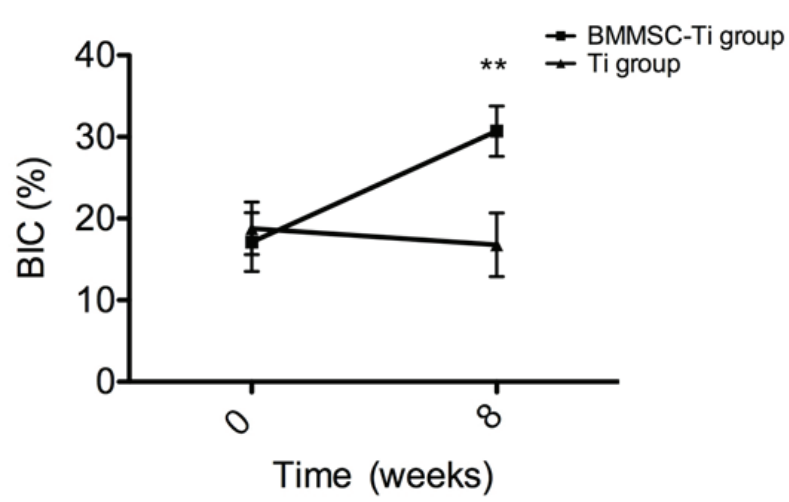

Figure 6. Histomorphometric analysis of BIC rate. After 8 weeks of bone healing in vivo, the average rate of BIC for BMSC sheet-implant complexes was greater compared with that for the unmodified Ti implants. ${ }^{* *} \mathrm{P}<0.001$. BIC, bone-implant contact; BMSC, bone marrow-derived mesenchymal stem cells; Ti, titanium.

osseointegration of dental implants is unpredictable and remains a clinical challenge $(2,30,31)$. Osteoporosis, one of 
A

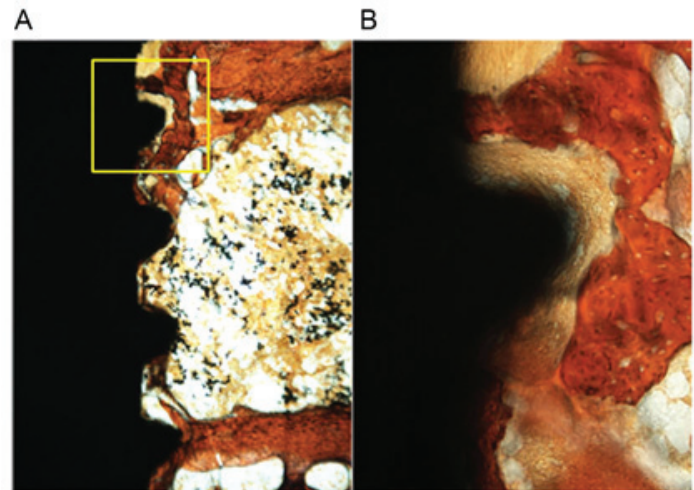

E

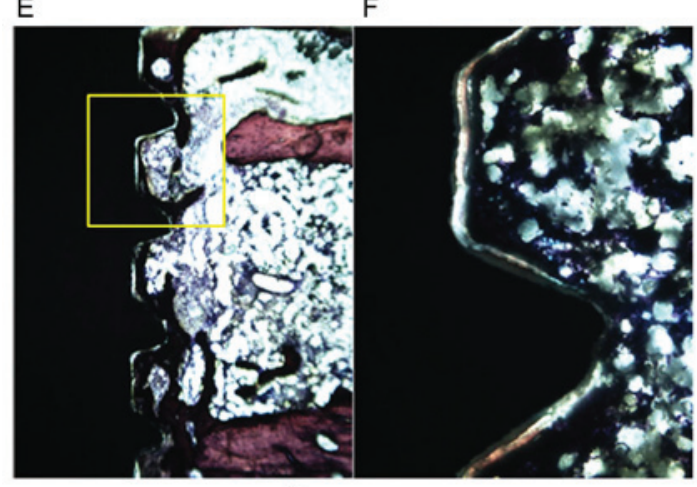

Ti group

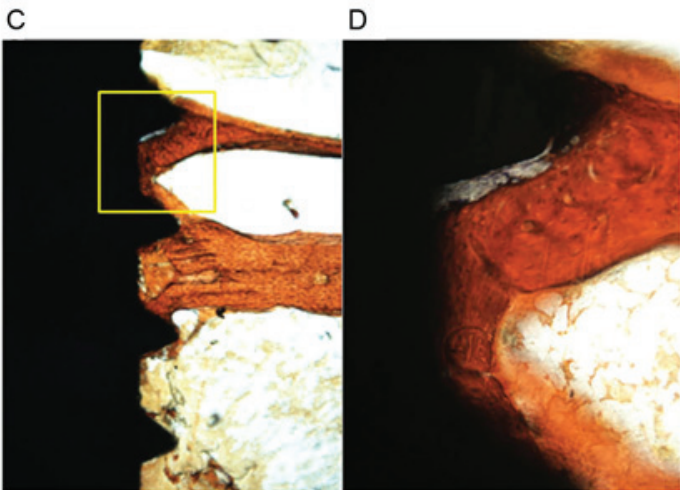

G $\quad H$

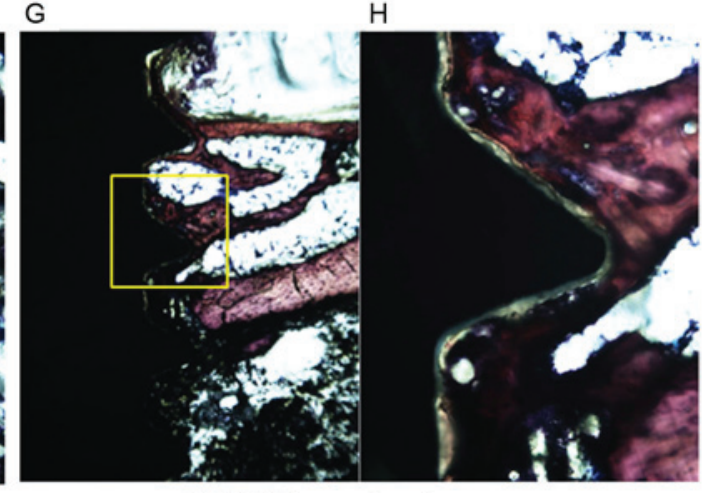

BMSC-Ti construct group

Figure 7. Histological evaluation of the bone-implant interface. After 8 weeks of bone healing in vivo, the tissues surrounding the implants were isolated for histological staining. Representative images of Ti and BMSC-Ti implant construct samples stained with (A-D) Masson-Ponceau Trichrome or (E-H) Van Gieson's stain are presented. B, D, F and $\mathrm{H}$ are x20 magnified images of the boxed regions in $\mathrm{A}, \mathrm{C}, \mathrm{E}$ and $\mathrm{H}$, respectively (original magnification, $\mathrm{x} 2.5$ ). Ti, titanium; BMSC, bone marrow-derived mesenchymal stem cells.

the most prevalent bone diseases, is principally caused by an imbalance between bone formation and resorption, resulting in the weaker structure of the host trabecular bone (32). In patients with osteoporosis, the success rate of dental implant treatment is primarily comprised of two factors: i) The bone density of the mandible and/or maxilla is reduced along with other parts of the skeleton; and ii) impaired bone metabolism in osteoporosis may reduce the healing capacity of bone around dental implants and prolong the healing process (33). To solve these problems, mutiple methods and ideas have been considered and proposed. Oral administrations of ibandronate or bisphosphonate are typically suggested for patients with osteoporosis, which are considered to be beneficial in increasing BMD and bone healing capacity. However, the final drug distribution in the alveolar bone and the effect on the bone healing ability around the implant remains unknown (34).

In our previous study, multilayer BMSC sheets, which were applied within the edentulous region, have showed positive effects on bone regeneration in the bone defect area (35). Moreover, the combination of the multilayer BMSC sheets and dental implant were proved to enhance the implant-bone osseointegration in both healthy and diabetic animals $(36,37)$. The improvement in the bone regeneration resulted from the BMSCs sheets probably lied in numerous cell factors released by BMSCs, including platelet-derived growth factors, bone morphogenetic protein-2, transforming growth factor- $\beta$ and insulin-like growth factor-I (38). These growth factors may enhance osteoblast differentiation and bone matrix mineralization (39). In addition, the superimposition of dense cell-ECM layers to form a 3D structure is currently being considered as a method of building tissues and scaffold materials in vitro for tissue regeneration (40). These 3D cell-based structures allow for a large number of cells and close cell-cell conjunctions. A study by Kii et al (41) demonstrated that a number of cadherins responsible for mediating cell-cell interactions, e.g. the M-cadherin and cadherin-11are associated with ECM stability. Therefore, a BMSC-implant structure that supports cell-to-cell interactions may also support osteogenic differentiation and proliferation (25). Furthermore, one more advantage of using the BMSC sheets was that the mechanical strength of the BMSCs sheets were relatively favorable, which could tolerate the friction during the surgical manipulation and insertion.

In the present study, the BMSC from healthy rats were used because it is a specific type of adult stem cell that differentiate into other cell types when a tissue has been lost and/or damaged due to trauma or disease, and their multilineage differentiation potential may be maintained during their lifespan. In addition, the BMSCs could also ensure the osteogenesis of transplanted cells. With the use of allogenic BMSCs in the sheet-implant constructs, no signs of rejection were observed during the experiment.

According to the micro-CT analysis and histologucal evaluation, at 8 weeks post-implantation, faster bone regeneration around BMSC-implant constructs was observed. Analysis of high-resolution 3D images obtained from 
micro-CT also demonstrated that all bone formation indices, including BV/TV, Tb.Th and BIC, were improved in newly formed areas around BMSC-implant constructs. Although the inherent biocompatibility of pure titanium implants also enables them to induce bone regeneration (42), the usage of multilayer BMSC sheets around titanium implants showed improved implant osseointegration, which may consequently improve the success rate of implant-based treatments in patients with low BMD.

Regarding the use of the present animal model, it may have been more appropriate and favorable to use larger OVX animal models in the present study, in which the alveolar bone would be a preferred choice for the installation of dental implant. Bone tissue in larger OVX animal models may exhibit closer similarities to humans regarding osteoporosis. However, the useage of live micro-CT scanning, which observed and analyzed the bone healing process atraumatically, limits the size of the sample being scanned. Therefore, the OVX rat model was adopted in the present study.

Further studies are required to investigate the combination of BMSCs with different cell types, such as periodontal ligament stem cells, dental pulp stem cells or gingiva-derived mesenchymal stem cells, which may produce higher levels of osteogenic factors or activate specific gene expression signaling pathways to release desirable osteogenic proteins. The BMSC-implant constructs should also be evaluated in different systematic diseases that interfere with bone healing in future studies.

In conclusion, the BMSC sheet-implant constructs improved the implant osseointegration in the rat model of osteoporosis. Micro-CT and histological analyses identified higher levels of BIC and new bone regeneration around BMSC-implant constructs. These results indicate that titanium implants combined with multilayer BMSC sheets may be considered in implant restoration for patients with osteoporosis on the basis of clinical trials.

\section{Acknowledgements}

The present study was partly supported by the Shaanxi provincial Science \& Technology Research Project, P.R.China (grant no. 2016SF-011).

\section{References}

1. Mendoza-Edroso C, Sánchez Garrido-Lestache N and Lopez-Picado A: Postmenopausal osteoporosis: Primary prevention or excessive use of medications. Semergen 39: 123-129, 2013 (In Spanish)

2. Esposito M, Hirsch JM, Lekholm U and Thomsen P: Biological factors contributing to failures of osseointegrated oral implants. (II). Etiopathogenesis. Eur J Oral Sci 106: 721-764, 1998.

3. Esposito M, Hirsch JM, Lekholm U and Thomsen P: Biological factors contributing to failures of osseointegrated oral implants. (I). Success criteria and epidemiology. Eur J Oral Sci 106: $527-551,1998$

4. Olivares-Navarrete R, Raines AL, Hyzy SL, Park JH, Hutton DL, Cochran DL, Boyan BD and Schwartz Z: Osteoblast maturation and new bone formation in response to titanium implant surface features are reduced with age. J Bone Miner Res 27: 1773-1783, 2012.

5. Shibli JA, Aguiar KC, Melo L, d'Avila S, Zenóbio EG, Faveri M, Iezzi $G$ and Piattelli A: Histological comparison between implants retrieved from patients with and without osteoporosis. Int J Oral Maxillofac Surg 37: 321-327, 2008.
6. Marco F, Milena F, Gianluca G and Vittoria O: Peri-implant osteogenesis in health and osteoporosis. Micron 36: 630-644, 2005.

7. Devlin H: Identification of the risk for osteoporosis in dental patients. Dent Clin North Am 56: 847-861, 2012.

8. Erdoğan O, Shafer DM, Taxel P and Freilich MA: A review of the association between osteoporosis and alveolar ridge augmentation. Oral Surg Oral Med Oral Pathol Oral Radiol Endod 104: 738.e1-13, 2007.

9. Blomqvist JE, Alberius P, Isaksson S, Linde A and Hansson BG: Factors in implant integration failure after bone grafting: An osteometric and endocrinologic matched analysis. Int J Oral Maxillofac Surg 25: 63-68, 1996.

10. de Melo L, Piattelli A, Lezzi G, d'Avila S, Zenóbio EG and Shibli JA: Human histologic evaluation of a six-year-old threaded implant retrieved from a subject with osteoporosis. J Contemp Dent Pract 9: 99-105, 2008

11. Shibli JA, Grande PA, d'Avila S, Iezzi G and Piattelli A: Evaluation of human bone around a dental implant retrieved from a subject with osteoporosis. Gen Dent 56: 64-67, 2008.

12. Thompson DD, Simmons HA, Pirie CM and Ke HZ: FDA Guidelines and animal models for osteoporosis. Bone (4 Suppl) 17: 125S-133S, 1995.

13. Ikebe K, Wada M, Kagawa $\mathrm{R}$ and Maeda Y: Is old age a risk factor for dental implants? Jpn Dent Sci Rev 45: 59-64, 2009.

14. Namkung-Matthai H, Appleyard R, Jansen J, Hao Lin J, Maastricht S, Swain M, Mason RS, Murrell GA, Diwan AD and Diamond T: Osteoporosis influences the early period of fracture healing in a rat osteoporotic model. Bone 28: 80-86, 2001.

15. Kalu DN: The ovariectomized rat model of postmenopausal bone loss. Bone Miner 15: 175-191, 1991.

16. Mosekilde L, Danielsen CC and Knudsen UB: The effect of aging and ovariectomy on the vertebral bone mass and biomechanical properties of mature rats. Bone 14: 1-6, 1993.

17. Yoshitake K, Yokota K, Kasugai Y, Kagawa M, Sukamoto T and Nakamura T: Effects of 16 weeks of treatment with tibolone on bone mass and bone mechanical and histomorphometric indices in mature ovariectomized rats with established osteopenia on a low-calcium diet. Bone 25: 311-319, 1999.

18. Vieira HP, Leite IA, Araújo Sampaio TM, Dos Anjos de Paula J, do Nascimento Andrade A, de Abreu LC, Valenti VE, Goulart FC and Adami F: Bisphosphonates adherence for treatment of osteoporosis. Int Arch Med 6: 24, 2013.

19. Le Guéhennec L, Soueidan A, Layrolle P and Amouriq Y: Surface treatments of titanium dental implants for rapid osseointegration. Dent Mater 23: 844-854, 2007.

20. Liu Y, Huse RO, de Groot K, Buser D and Hunziker EB: Delivery mode and efficacy of BMP-2 in association with implants. J Dent Res 86: 84-89, 2007.

21. Liu Y, Wu J, Zhu Y and Han J: Therapeutic application of mesenchymal stem cells in bone and joint diseases. Clin Exp Med 14: 13-24, 2014.

22. Nelson TJ, Behfar A, Yamada S, Martinez-Fernandez A and Terzic A: Stem cell platforms for regenerative medicine. Clin Transl Sci 2: 222-227, 2009.

23. Pino AM, Rosen CJ and Rodríguez JP: In osteoporosis, differentiation of mesenchymal stem cells (MSCs) improves bone marrow adipogenesis. Biol Res 45: 279-287, 2012.

24. Yamato $\mathrm{M}$ and Okano T: Cell sheet engineering. Mater Today 7 : 42-47, 2004.

25. Zhou W, Han C, Song Y, Yan X, Li D, Chai Z, Feng Z, Dong Y, Li L, Xie X, et al: The performance of bone marrow mesenchymal stem cell-implant complexes prepared by cell sheet engineering techniques. Biomaterials 31: 3212-3221, 2010.

26. Mandal BB and Kundu SC: Osteogenic and adipogenic differentiation of rat bone marrow cells on non-mulberry and mulberry silk gland fibroin 3D scaffolds. Biomaterials 30: 5019-5030, 2009.

27. Agata H, Yamazaki M, Uehara M, Hori A, Sumita Y, Tojo A and Kagami H: Characteristic differences among osteogenic cell populations of rat bone marrow stromal cells isolated from untreated, hemolyzed or Ficoll-treated marrow. Cytotherapy 14: 791-801, 2012.

28. Apgar JM, Juarranz A, Espada J, Villanueva A, Cañete M and Stockert JC: Fluorescence microscopy of rat embryo sections stained with haematoxylin-eosin and Masson's trichrome method. J Microsc 191: 20-27, 1998.

29. Sykaras N, Iacopino AM, Marker VA, Triplett RG and Woody RD: Implant materials, designs, and surface topographies: Their effect on osseointegration. A literature review. Int J Oral Maxillofac Implants 15: 675-690, 2000. 
30. Mombelli A and Cionca N: Systemic diseases affecting osseointegration therapy. Clin Oral Implants Res 17 (Suppl 2): S97-S103, 2006.

31. Porter JA and von Fraunhofer JA: Success or failure of dental implants? A literature review with treatment considerations. Gen Dent 53: 423-432; quiz 433, 446, 2005.

32. Muñoz-Torres M, Alonso G and Raya MP: Calcitonin therapy in osteoporosis. Treat Endocrinol 3: 117-132, 2004.

33. Dao TT, Anderson JD and Zarb GA: Is osteoporosis a risk factor for osseointegration of dental implants? Int J Oral Maxillofac Implants 8: 137-144, 1993.

34. Recknor C, Czerwinski E, Bone HG, Bonnick SL, Binkley N, Palacios S, Moffett A, Siddhanti S, Ferreira I, Ghelani P, et al: Denosumab compared with ibandronate in postmenopausal women previously treated with bisphosphonate therapy: A randomized open-label trial. Obstet Gynecol 121: 1291-1299,2013.

35. Liu Y, Ming L, Luo H, Liu W, Zhang Y, Liu H and Jin Y: Integration of a calcined bovine bone and BMSC-sheet 3D scaffold and the promotion of bone regeneration in large defects. Biomaterials 34: 9998-10006, 2013.

36. Xu B, Zhang J, Brewer E, Tu Q, Yu L, Tang J, Krebsbach P, Wieland $\mathrm{M}$ and Chen J: Osterix enhances BMSC-associated osseointegration of implants. J Dent Res 88: 1003-1007, 2009.
37. Kotsovilis S, Karoussis IK and Fourmousis I: A comprehensive and critical review of dental implant placement in diabetic animals and patients. Clin Oral Implants Res 17: 587-599, 2006.

38. Lalani Z, Wong M, Brey EM, Mikos AG and Duke PJ: Spatial and temporal localization of transforming growth factor-beta1, bone morphogenetic protein-2, and platelet-derived growth factor-A in healing tooth extraction sockets in a rabbit model. J Oral Maxillofac Surg 61: 1061-1072, 2003.

39. Yamada Y, Ueda M, Naiki T and Nagasaka T: Tissue-engineered injectable bone regeneration for osseointegrated dental implants. Clin Oral Implants Res 15: 589-597, 2004.

40. Gkantidis N, Schauseil M, Pazera P, Zorkun B, Katsaros C and Ludwig B: Evaluation of 3-dimensional superimposition techniques on various skeletal structures of the head using surface models. PloS One 10: e0118810, 2015.

41. Kii I, Amizuka N, Shimomura J, Saga Y and Kudo A: Cell-cell interaction mediated by cadherin-11 directly regulates the differentiation of mesenchymal cells into the cells of the osteo-lineage and the chondro-lineage. J Bone Miner Res 19: 1840-1849, 2004.

42. Oron A, Agar G, Oron U and Stein A: Correlation between rate of bony ingrowth to stainless steel, pure titanium, and titanium alloy implants in vivo and formation of hydroxyapatite on their surfaces in vitro. J Biomed Mater Res A 91: 1006-1009, 2009. 